\title{
Contiguous Function Relations for $k$-Hypergeometric Functions
}

\author{
Shahid Mubeen, Gauhar Rahman, Abdur Rehman, and Mammona Naz
}

Department of Mathematics, University of Sargodha, Sargodha 40100, Pakistan

Correspondence should be addressed to Gauhar Rahman; gauhar55uom@gmail.com

Received 4 December 2013; Accepted 27 January 2014; Published 10 April 2014

Academic Editors: Y. S. Han and S. Zhang

Copyright (C) 2014 Shahid Mubeen et al. This is an open access article distributed under the Creative Commons Attribution License, which permits unrestricted use, distribution, and reproduction in any medium, provided the original work is properly cited.

\begin{abstract}
In this research work, our aim is to determine the contiguous function relations for $k$-hypergeometric functions with one parameter corresponding to Gauss fifteen contiguous function relations for hypergeometric functions and also obtain contiguous function relations for two parameters. Throughout in this research paper, we find out the contiguous function relations for both the cases in terms of a new parameter $k>0$. Obviously if $k \rightarrow 1$, then the contiguous function relations for $k$-hypergeometric functions are Gauss contiguous function relations.
\end{abstract}

\section{Introduction}

The hypergeometric function ${ }_{2} F_{1}(a, b ; c ; z)$ plays an important role in mathematical analysis and its applications. This function allows us to solve many interesting problems, such as conformal mapping of triangular domains bounded by line segments or circular arcs and various problems of quantum mechanics. Most of the functions that occur in the analysis are special cases of the hypergeometric functions. Gauss first introduced and studied hypergeometric series, paying special attention to the cases when a series can be summed into an elementary function. This gives one of the motivations for studying hypergeometric series; that is, the fact that the elementary functions and several other important functions in mathematics can be expressed in terms of hypergeometric functions. Hypergeometric functions can also be described as solutions of special second order linear differential equations, the hypergeometric differential equations. Riemann was the first to exploit this idea and introduce a special symbol to classify hypergeometric functions by singularities and exponents of differential equations. The hypergeometric function is a solution of the following Euler's hypergeometric differential equation $z(1-z)\left(d^{2} \omega / d z^{2}\right)+[c-(a+b+1) z](d \omega / d z)-a b \omega=$ 0 , which has three regular singular points 0,1 , and $\infty$. The generalization of this equation to three arbitrary regular singular points is given by Riemann's differential equation. Any second order differential equation with three regular singular points can be converted to the hypergeometric differential equation by changing of variable.
On the other hand, in theory of hypergeometric function, the terminology contiguous function was introduced for the case in which one of the parameters is shifted by \pm 1 . For example, $F(a+1, b ; c ; z)$ is contiguous to $F(a, b ; c ; z)$. Gauss defined two hypergeometric functions to be contiguous if they have the same power series variable and if two of the parameters are pairwise equal and if third pair differs by \pm 1 . He proved that between ${ }_{2} F_{1}(a, b ; c ; z)$ and any two of its contiguous functions, there exists a linear relation with coefficients at most linear in $z$. These relationships are of great use in extending numerical tables of the function, since, for one fixed value of $z$, it is necessary only to calculate the value of the function over two units in $a, b$, and $c$, and apply some recurrence relations in order to find the function values over a large range of values of $a, b$, and $c$ in this particular $z$-plane. Since there are six contiguous functions to a given function ${ }_{2} F_{1}$, Gauss [1] got a total of fifteen relations. In fact, only four of the fifteen are really independent as all others may be obtained by elimination and use of the fact that the ${ }_{2} F_{1}$ is symmetric in $a$ and $b$.

Applications of the contiguous function relations range from the evaluation of hypergeometric series to the derivation of the summation and transformation formulas for such series; these can be used to evaluate the contiguous functions to a hypergeometric function. For this, in a series of three research papers, Lavoie et al. [2,3] have obtained a large number of very interesting results contiguous to Gauss second, Kummer, and Bailey theorems for the series ${ }_{2} F_{1}$. For more details about hypergeometric series and their contiguous relations, see [4-10]. 
In 1996, Morita [11] used Gauss contiguous relations in computing the hypergeometric function ${ }_{2} F_{1}[n+1 / 2, n+$ $1 / 2 ; m ; z]$. In 1996, Gupta et al. [12] derived contiguous relations, basic hypergeometric functions, and orthogonal polynomials. In 2002, Vidunas [13] generalized the Kummer identity by using the contiguous relations. In 2003, Vidunas [14] defined several properties of coefficients of these general contiguous relations and then used them to propose effective ways to compute contiguous relations. In 2006, Rakha and Ibrahim [15] obtained some interesting consequences of the contiguous relations of ${ }_{2} F_{1}$. In 2008, Ibrahim and Rakha [16] derived the contiguous relations and their computations for ${ }_{2} F_{1}$ hypergeometric series. They obtained the interesting formula as a linear relation of three shifted Gauss polynomials in the parameters $a, b$, and $c$.

Díaz et al. [17-19] introduced $k$-gamma and $k$-beta functions $(k>0)$ and proved a number of their properties. They have also studied $k$-zeta functions and $k$-hypergeometric functions based on Pochhammer $k$-symbols for factorial functions. Very recently, many researchers [20-23] followed the work of Díaz et al., they studied and obtained some consequence results of $k$-beta, $k$-gamma, $k$-zeta, and $k$-hypergeometric functions and their properties. In 2009, Mansour [24] obtained the $k$-generalized gamma function $\Gamma_{k}(x)$ by functional equations. In 2012, Mubeen and Habibullah [25] defined $k$-fractional integration and gave its applications regarding fractional integrals. In 2012, Mubeen and Habibullah [26] also gave a useful and simple integral representation of some confluent $k$-hypergeometric functions ${ }_{1} F_{1, k}$ and $k$-hypergeometric functions ${ }_{2} F_{1, k}$. Furthermore, in 2013, Mubeen [27] defined a second order linear $k$-hypergeometric differential equation $k z(1-k z) \omega^{\prime \prime}+[c-(a+b+k) k z] \omega^{\prime}-$ $a b \omega=0$ having one solution in the form of $k$-hypergeometric function ${ }_{2} F_{1, k}(a, b ; c ; z)$.

\section{Basic Concepts}

In this section, we present the definitions of some basic concepts in the term of new parameter $k>0$.

Definition 1. Two hypergeometric functions are said to be contiguous if their parameters $a, b$, and $c$ differ by integers. The relations made by contiguous functions are said to be contiguous function relations.

Definition 2. Let $k>0$; then the Pochhammer $k$-symbol is defined by $(a)_{n, k}=a(a+k)(a+2 k) \cdots(a+(n-1) k)$, for $n \geq 1$, $a \neq 0$, and $(a)_{0, k}=0$.

Definition 3. For $k>0$ and $z \in \mathbb{C}$, the $k$-gamma function $\Gamma_{k}$ is defined by

$$
\Gamma_{k}(z)=\lim _{n \rightarrow \infty} \frac{n ! k^{n}(n k)^{z / k-1}}{(z)_{n, k}} .
$$

Its integral representation is also given by

$$
\Gamma_{k}(z)=\int_{0}^{\infty} t^{z-1} e^{-t^{k} / k} d t
$$

The relation between Pochhammer $k$-symbol and $k$-gamma function is given as follows:

$$
(z)_{n, k}=\frac{\Gamma_{k}(z+n k)}{\Gamma_{k}(z)} .
$$

Furthermore, we can write $k$-gamma function in terms of ordinary gamma function in the following form:

$$
\Gamma_{k}(z)=k^{z / k-1} \Gamma\left(\frac{z}{k}\right) .
$$

Definition 4. The $k$-hypergeometric function with three parameters $a, b$, and $c$, two parameters $a, b$ in numerator and one parameter $c$ in denominator is defined by

$$
{ }_{2} F_{1, k}(a, b ; c ; z)=\sum_{n=0}^{\infty} \frac{(a)_{n, k}(b)_{n, k} z^{n}}{(c)_{n, k} n !}
$$

for all $a, b, c \in \mathbb{C}, c \neq 0,-1,-2,-3, \ldots,|z|<1$, and $k>0$.

\section{Contiguous Functions for $k$-Hypergeometric Function with One Parameter}

If we increase or decrease one and only one of the parameters of $k$-hypergeometric function,

$$
{ }_{2} F_{1, k}(a, b ; c ; z)=\sum_{n=0}^{\infty} \frac{(a)_{n, k}(b)_{n, k} z^{n}}{(c)_{n, k} n !}
$$

by $\pm k$, then the resultant function is said to be contiguous to ${ }_{2} F_{1, k}$. For simplicity, we use the following notations:

$$
\begin{gathered}
F_{k}=F_{k}(a, b ; c ; z)=\sum_{n=0}^{\infty} \frac{(a)_{n, k}(b)_{n, k} z^{n}}{(c)_{n, k} n !}, \\
F_{k}(a+)=F_{k}(a+k, b ; c ; z)=\sum_{n=0}^{\infty} \frac{(a+k)_{n, k}(b)_{n, k} z^{n}}{(c)_{n, k} n !}, \\
F_{k}(a-)=F_{k}(a-k, b ; c ; z)=\sum_{n=0}^{\infty} \frac{(a-k)_{n, k}(b)_{n, k} z^{n}}{(c)_{n, k} n !} .
\end{gathered}
$$

Similarly, we can write the notations for $F_{k}(b+), F_{k}(b-)$, $F_{k}(c+)$, and $F_{k}(c-)$.

Let

$$
\delta_{n, k}=\frac{(a)_{n, k}(b)_{n, k} z^{n}}{(c)_{n, k} n !} .
$$

Then, (7) becomes

$$
F_{k}=\sum_{n=0}^{\infty} \delta_{n, k} .
$$

Now, consider (8) as

$$
F_{k}(a+)=\sum_{n=0}^{\infty} \frac{(a+k)_{n, k}(b)_{n, k} z^{n}}{(c)_{n, k} n !} .
$$


Since $a(a+k)_{n, k}=(a)_{n, k}(a+n k)$, therefore, by using this result in (12), we obtain

$$
F_{k}(a+)=\sum_{n=0}^{\infty} \frac{(a+n k) \delta_{n, k}}{a} .
$$

Similarly, we can write $(9)$ by using the result $(a+(n-1) k)(a-$ $k)_{n, k}=(a-k)(a)_{n, k}$ as

$$
F_{k}(a-)=\sum_{n=0}^{\infty} \frac{(a-k) \delta_{n, k}}{(a+(n-1) k)} .
$$

Thus we have the following six contiguous functions for ${ }_{2} F_{1, k}$, where $k>0$ :

$$
\begin{gathered}
F_{k}(a+)=\sum_{n=0}^{\infty} \frac{(a+n k) \delta_{n, k}}{a}, \\
F_{k}(a-)=\sum_{n=0}^{\infty} \frac{(a-k) \delta_{n, k}}{(a+(n-1) k)}, \\
F_{k}(b+)=\sum_{n=0}^{\infty} \frac{(b+n k) \delta_{n, k}}{b}, \\
F_{k}(b-)=\sum_{n=0}^{\infty} \frac{(b-k) \delta_{n, k}}{(b+(n-1) k)}, \\
F_{k}(c+)=\sum_{n=0}^{\infty} \frac{(c) \delta_{n, k}}{(c+n k)}, \\
F_{k}(c-)=\sum_{n=0}^{\infty} \frac{(c+(n-1) k) \delta_{n, k}}{(c-k)} .
\end{gathered}
$$

By the help of differential operator $k \theta=k z(d / d z)$, we get the following result:

$$
(k \theta+a) F_{k}=(k \theta+a) \sum_{n=0}^{\infty} \frac{(a)_{n, k}(b)_{n, k} z^{n}}{(c)_{n, k} n !} .
$$

Hence, with the aid of (12), it follows that

$$
(k \theta+a) F_{k}=a F_{k}(a+) .
$$

Similarly, we can also write the following relations as

$$
\begin{gathered}
(k \theta+b) F_{k}=b F_{k}(b+), \\
(k \theta+c-k) F_{k}=(c-k) F_{k}(c-) .
\end{gathered}
$$

\section{Contiguous Relations for $k$-Hypergeometric Function}

Since there are six contiguous functions to a given function ${ }_{2} F_{1, k}$, therefore, we have to obtain the following fifteen contiguous function relations for $k$-hypergeometric function ${ }_{2} F_{1, k}$, for all $a, b, c \in \mathbb{C}$ and $k>0$. In fact, only four of the fifteen are really independent as all others may be obtained by elimination and use of the fact that the ${ }_{2} F_{1, k}$ is symmetric in $a$ and $b$.
Relation 1. Consider

$$
(a-b) F_{k}=a F_{k}(a+)-b F_{k}(b+) .
$$

Proof. By subtracting (18) from (17), we get

$$
(k \theta+a-k \theta-b)=a F_{k}(a+)-b F_{k}(b+) .
$$

This implies the required relation.

Relation 2. Consider

$$
(a-c+k) F_{k}=a F_{k}(a+)-(c-k) F_{k}(c-) .
$$

Proof. By subtracting (19) from (17), we obtain

$$
\begin{gathered}
(k \theta+a) F_{k}-(k \theta+c-k) F_{k}=a F_{k}-(c-k) F_{k}(c-) \\
\Longrightarrow(a-c+k) F_{k}=a F_{k}(a+)-(c-k) F_{k}(c-) .
\end{gathered}
$$

Relation 3. Consider

$$
\begin{aligned}
{[a} & +(b-c) k z] F_{k} \\
& =a(1-k z) F_{k}(a+)-c^{-1}(c-a)(c-b) k z F_{k}(c+) .
\end{aligned}
$$

Proof. Let us consider

$$
F_{k}=\sum_{n=0}^{\infty} \frac{(a)_{n, k}(b)_{n, k} z^{n}}{(c)_{n, k} n !} .
$$

By the help of differential operator $k \theta=k z(d / d z)$, we get the following:

$$
k \theta F_{k}=\sum_{n=1}^{\infty} \frac{k(a)_{n, k}(b)_{n, k} z^{n}}{(c)_{n, k}(n-1) !} .
$$

By shifting the index $n$ with $n+1$, we get

$$
k \theta F_{k}=k \sum_{n=0}^{\infty} \frac{(a)_{n+1, k}(b)_{n+1, k} z^{n+1}}{(c)_{n+1, k} n !} .
$$

Since $(a)_{n+1, k}=(a)_{n, k}(a+n k),(b)_{n+1, k}=(b)_{n, k}(b+n k)$, and $(c)_{n+1, k}=(c)_{n, k}(c+n k)$, therefore, by putting these three results in (27), we have

$$
k \theta F_{k}=k z \sum_{n=0}^{\infty} \frac{(a+n k)(b+n k) \delta_{n, k}}{(c+n k)} .
$$

Since $(a+n k)(b+n k) /(c+n k)=n k+(a+b-c)+(c-a)(c-$ $b) /(c+n k)$, by substituting this result in (28), we obtain

$$
\begin{aligned}
k \theta F_{k}= & k z \sum_{n=0}^{\infty} n k \delta_{n, k}+(a+b-c) k z \sum_{n=0}^{\infty} \delta_{n, k} \\
& +c^{-1}(c-a)(c-b) k z \sum_{n=0}^{\infty} \frac{c \delta_{n, k}}{c+n k} \\
= & k^{2} z \theta F_{k}+(a+b-c) k z F_{k} \\
& +c^{-1}(c-a)(c-b) k z F_{k}(c+) .
\end{aligned}
$$


This implies that

$$
\begin{aligned}
& (1-k z) k \theta F_{k} \\
& \quad=(a+b-c) k z F_{k}+c^{-1}(c-a)(c-b) k z F_{k}(c+) .
\end{aligned}
$$

Now, from (17), we have

$$
(1-k z) k \theta F_{k}=-a(1-k z) F_{k}+a(1-k z) F_{k}(a+) .
$$

By comparing these two equations, (30) and (31), we get

$$
\begin{aligned}
{[a} & +(b-c) k z] F_{k} \\
& =a(1-k z) F_{k}(a+)-c^{-1}(c-a)(c-b) k z F_{k}(c+) .
\end{aligned}
$$

Relation 4. Consider

$$
(1-k z) F_{k}=F_{k}(a-)-c^{-1}(c-b) k z F_{k}(c+) \text {. }
$$

Proof. Let us consider

$$
F_{k}(a-)=\sum_{n=0}^{\infty} \frac{(a-k)_{n, k} \delta_{n, k}}{(a)_{n, k}} .
$$

By applying the differential operator $k \theta=k z(d / d z)$, we get

$$
k \theta F_{k}(a-)=\sum_{n=1}^{\infty} \frac{k(a-k)_{n, k}(b)_{n, k} z^{n}}{(c)_{n, k}(n-1) !} .
$$

By shifting the index $n$ with $n+1$, we can write

$$
k \theta F_{k}(a-)=k \sum_{n=0}^{\infty} \frac{(a-k)_{n+1, k}(b)_{n+1, k} z^{n+1}}{(c)_{n+1, k} n !} .
$$

Since $(a-k)_{n+1, k}=(a-k)(a)_{n, k},(b)_{n+1, k}=(b+n k)(b)_{n, k}$, and $(c)_{n+1, k}=(c+n k)(c)_{n, k}$, by substituting these three results in (36), we obtain the following:

$$
\begin{aligned}
k \theta F_{k}(a-) & =k \sum_{n=0}^{\infty} \frac{(a-k)(a)_{n, k}(b+n k)(b)_{n, k} z^{n+1}}{(c+n k)(c)_{n, k} n !} \\
& =(a-k) k z \sum_{n=0}^{\infty} \delta_{n, k}-\frac{(a-k)(c-b)}{(c+n k)} k z \sum_{n=0}^{\infty} \delta_{n, k} .
\end{aligned}
$$

This implies that

$$
k \theta F_{k}(a-)=(a-k) k z F_{k}-c^{-1}(a-k)(c-b) k z F_{k}(c+) .
$$

Now, by replacing $a$ with $a-k$ in (17), we get

$$
k \theta F_{k}(a-)=(a-k) F_{k}-(a-k) F_{k}(a-) .
$$

By comparing (38) and (39), we get

$$
(1-k z) F_{k}=F_{k}(a-)-c^{-1}(c-b) k z F_{k}(c+) .
$$

Now, we obtain the remaining contiguous function relations by elimination and use of the fact that the ${ }_{2} F_{1, k}$ is symmetric in $a$ and $b$, for $k>0$.
Relation 5. Consider

$$
(1-k z) F_{k}=F_{k}(b-)-c^{-1}(c-a) k z F_{k}(c+) .
$$

Relation 6. Consider

$$
[2 a-c+(b-a) k z] F_{k}=a(1-k z) F_{k}(a+)-(c-a) F_{k}(a-) .
$$

Relation 7. Consider

$$
(a+b-c) F_{k}=a(1-k z) F_{k}(a+)-(c-b) F_{k}(b-) .
$$

Relation 8. Consider

$$
(c-a-b) F_{k}=(c-a) F_{k}(a-)-b(1-k z) F_{k}(b+) .
$$

Relation 9. Consider

$$
(b-a)(1-k z) F_{k}=(c-a) F_{k}(a-)-(c-b) F_{k}(b-) \text {. }
$$

Relation 10. Consider

$$
\begin{aligned}
& {[k-a+(c-b-k) k z] F_{k}} \\
& \quad=(c-a) F_{k}(a-)-(c-k)(1-k z) F_{k}(c-) .
\end{aligned}
$$

Relation 11. Consider

$$
[2 b-c+(a-b) k z] F_{k}=b(1-k z) F_{k}(b+)-(c-b) F_{k}(b-) \text {. }
$$

Relation 12. Consider

$$
\begin{aligned}
{[b} & +(a-c) k z] F_{k} \\
& =b(1-k z) F_{k}(b+)-c^{-1}(c-a)(c-b) k z F_{k}(c+) .
\end{aligned}
$$

Relation 13. Consider

$$
(b-c+k) F_{k}=b F_{k}(b+)-(c-k) F_{k}(c-) .
$$

Relation 14. Consider

$$
\begin{aligned}
& {[k-b+(c-a-k) k z] F_{k}} \\
& \quad=(c-b) F_{k}(b-)-(c-k)(1-k z) F_{k}(c-) .
\end{aligned}
$$

Relation 15. Consider

$$
\begin{aligned}
{[c-k+(a+b+k-2 c) k z] F_{k} } \\
\quad=(c-k)(1-k z) F_{k}(c-)-c^{-1}(c-a)(c-b) k z F_{k}(c+) .
\end{aligned}
$$




\section{Contiguous Functions for $k$-Hypergeometric Function with Two Parameters}

In this section, we obtain contiguous functions for $k$-hypergeometric function with two parameters. Since we know $F_{k}(a+)=\sum_{n=0}^{\infty}\left((a+k)_{n, k} \delta_{n, k} /(a)_{n, k}\right), F_{k}(a-)=\sum_{n=0}^{\infty}((a-$ $\left.k)_{n, k} \delta_{n, k} /(a)_{n, k}\right)$, and $F_{k}(b+)=\sum_{n=0}^{\infty}\left((b+k)_{n, k} \delta_{n, k} /(b)_{n, k}\right)$, where $\delta_{n, k}=(a)_{n, k}(b)_{n, k} z^{n} /(c)_{n, k} n$ !, therefore, by using these contiguous functions, we obtain the following contiguous function with two parameters

$$
\begin{aligned}
F_{k}(a+, b+) & =F_{k}(a+k, b+k ; c ; z) \\
& =\sum_{n=0}^{\infty} \frac{(a+k)_{n, k}(b+k)_{n, k} z^{n}}{(c)_{n, k} n !} .
\end{aligned}
$$

Similarly, we can write

$$
\begin{gathered}
F_{k}(a+, c+)=F_{k}(a+k, b ; c+k ; z)=\sum_{n=0}^{\infty} \frac{(a+k)_{n, k}(b)_{n, k} z^{n}}{(c+k)_{n, k} n !} \\
\begin{aligned}
F_{k}(a-, b-) & =F_{k}(a-k, b-k ; c ; z) \\
& =\sum_{n=0}^{\infty} \frac{(a-k)_{n, k}(b-k)_{n, k} z^{n}}{(c)_{n, k} n !} .
\end{aligned}
\end{gathered}
$$

Now, we have to prove the following contiguous relations.

Relation 16. It show that

$$
F_{k}(a-)-F_{k}(b-)+c^{-1}(b-a) k z F_{k}(c+)=0 .
$$

Proof. One has to prove that

$$
F_{k}(a-)-F_{k}(b-)+c^{-1}(b-a) k z F_{k}(c+)=0 .
$$

From Relations 4 and 5, respectively, we have

$$
\begin{aligned}
& F_{k}(a-)=(1-k z) F_{k}+c^{-1}(c-b) k z F_{k}(c+), \\
& F_{k}(b-)=(1-k z) F_{k}+c^{-1}(c-a) k z F_{k}(c+) .
\end{aligned}
$$

Therefore, by substituting the values of $F_{k}(a-)$ and $F_{k}(b-)$ in (55), we get

$$
\begin{aligned}
F_{k}(a-) & -F_{k}(b-)+c^{-1}(b-a) k z F_{k}(c+) \\
= & (1-k z) F_{k}+c^{-1}(c-b) k z F_{k}(c+)-(1-k z) F_{k} \\
& -c^{-1}(c-a) k z F_{k}(c+)+c^{-1}(b-a) k z F_{k}(c+) .
\end{aligned}
$$

After simplification, we get the required relation. Consider

$$
F_{k}(a-)-F_{k}(b-)+c^{-1}(b-a) k z F_{k}(c+)=0 .
$$

Relation 17. It shows that

$$
F_{k}=F_{k}(a-, b+)+c^{-1}(b+k-a) k z F_{k}(b+, c+) .
$$

Proof. From Relation 16, we have

$$
F_{k}(b-)=F_{k}(a-)+c^{-1}(b-a) k z F_{k}(c+) .
$$

This can be written as

$$
\begin{aligned}
& F_{k}(a, b-k ; c ; z) \\
& \quad=F_{k}(a-k, b ; c ; z)+c^{-1}(b-a) k z F_{k}(a, b ; c+k ; z) .
\end{aligned}
$$

Therefore, by replacing $b$ with $b+k$ in (61), we obtain

$$
F_{k}=F_{k}(a-, b+)+c^{-1}(b+k-a) k z F_{k}(b+, c+) \text {. }
$$

Relation 18. It shows that

$$
\begin{aligned}
& (c-k-b) F_{k} \\
& \quad=(c-a) F_{k}(a-, b+)+(a-k-b)(1-k z) F_{k}(b+, c) .
\end{aligned}
$$

Proof. By consider Relation 9,

$$
(b-a)(1-k z) F_{k}=(c-a) F_{k}(a-)-(c-b) F_{k}(b-) .
$$

This may be written as

$$
\begin{aligned}
& (b-a)(1-k z) F_{k}(a, b ; c ; z) \\
& \quad=(c-a) F_{k}(a-k, b ; c ; z)-(c-b) F_{k}(a, b-k ; c ; z) .
\end{aligned}
$$

Now, by replacing $b$ with $b+k$ in Relation 9 , we obtain

$$
\begin{aligned}
(c-k-b) F_{k} & \\
& =(c-a) F_{k}(a-, b+)+(a-k-b)(1-k z) F_{k}(b+, c) .
\end{aligned}
$$

\section{Conclusion}

In this research work, we determined the contiguous function relations for $k$-hypergeometric function with one and two parameters. So we conclude that if $k \rightarrow 1$, we obtain Gauss and Rainville contiguous function relations for hypergeometric functions.

\section{Conflict of Interests}

The authors declare that there is no conflict of interests regarding the publication of this paper.

\section{Acknowledgments}

The authors would like to express profound gratitude to referees for their deeper review of this paper and the referee's useful suggestions that led to an improved presentation of the paper. 


\section{References}

[1] E. D. Rainville, Special Functions, The Macmillan, New York, NY, USA, 1960.

[2] J.-L. Lavoie, F. Grondin, and A. K. Rathie, "Generalizations of Watson's theorem on the sum of $\mathrm{a}_{3} F_{2}$," Indian Journal of Mathematics, vol. 34, no. 1, pp. 23-32, 1992.

[3] J. L. Lavoie, F. Grondin, and A. K. Rathie, "Generalizations of Whipple's theorem on the sum of $\mathrm{a}_{3} F_{2}$," Journal of Computational and Applied Mathematics, vol. 72, no. 2, pp. 293-300, 1996.

[4] M. Abramowitz and I. Stegun, Handbook of Mathematical Functions with Formulas, Graphs, and Mathematical Tables, Dover, New York, NY, USA, 1972.

[5] P. Agarwal, "Contiguous relations for bilateral basic hypergeometric series," International Journal of Mathematical Sciences, vol. 3, no. 2, pp. 375-388, 2004.

[6] G. E. Andrews, R. Askey, and R. Roy, Special Functions, Cambridge University Press, Cambridge, UK, 1999.

[7] G. Gasper and M. Rahman, Basic Hypergeometric Series, Encyclopedia of Mathematics and its Applications, Cambridge University Press, Cambridge, UK, 1990.

[8] D. P. Gupta and D. R. Masson, "Contiguous relations, continued fractions and orthogonality," Transactions of the American Mathematical Society, vol. 350, no. 2, pp. 769-808, 1998.

[9] M. E. H. Ismail and C. A. Libis, "Contiguous relations, basic hypergeometric functions, and orthogonal polynomials. I," Journal of Mathematical Analysis and Applications, vol. 141, no. 2, pp. 349-372, 1989.

[10] E. D. Rainville, "The contiguous function relations for ${ }_{p} F_{q}$ with applications to Bateman's $J_{u, v}^{n}$ and Rice's $H_{n}(\zeta ; \rho ; v)$," Bulletin of the American Mathematical Society, vol. 51, pp. 714-723, 1945.

[11] T. Morita, "Use of the gauss contiguous relations in computing the hypergeometric functions ${ }_{2} F_{1}(n+1 / 2, n+1 / 2 ; m ; z)$," Interdisciplinary Information Sciences, vol. 2, no. 1, pp. 63-74, 1996.

[12] D. P. Gupta, M. E. H. Ismail, and D. R. Masson, "Contiguous relations, basic hypergeometric functions, and orthogonal polynomials. III. Associated continuous dual $q$-Hahn polynomials," Journal of Computational and Applied Mathematics, vol. 68, no. 1-2, pp. 115-149, 1996.

[13] R. Vidunas, “A generalization of Kummer's identity," The Rocky Mountain Journal of Mathematics, vol. 32, no. 2, pp. 919-936, 2002.

[14] R. Vidunas, "Contiguous relations of hypergeometric series," Journal of Mathematical Analysis and Applications, vol. 135, pp. 507-519, 2003.

[15] M. A. Rakha and A. K. Ibrahim, "On the contiguous relations of hypergeometric series," Journal of Computational and Applied Mathematics, vol. 192, no. 2, pp. 396-410, 2006.

[16] A. K. Ibrahim and M. A. Rakha, "Contiguous relations and their computations for ${ }_{2} F_{1}$ hypergeometric series," Computers \& Mathematics with Applications, vol. 56, no. 8, pp. 1918-1926, 2008.

[17] R. Díaz and C. Teruel, " $q$, $k$-generalized gamma and beta functions," Journal of Nonlinear Mathematical Physics, vol. 12, no. 1, pp. 118-134, 2005.

[18] R. Díaz and E. Pariguan, "On hypergeometric functions and Pochhammer k-symbol," Divulgaciones Matemáticas, vol. 15, no. 2, pp. 179-192, 2007.

[19] R. Díaz, C. Ortiz, and E. Pariguan, "On the $k$-gamma $q$-distribution," Central European Journal of Mathematics, vol. 8, no. 3, pp. $448-458,2010$.
[20] C. G. Kokologiannaki, "Properties and inequalities of generalized $k$-gamma, beta and zeta functions," International Journal of Contemporary Mathematical Sciences, vol. 5, no. 13-16, pp. 653660, 2010.

[21] V. Krasniqi, "A limit for the $k$-gamma and $k$-beta function," International Mathematical Forum, vol. 5, no. 33, pp. 1613-1617, 2010.

[22] V. Krasniqi, "Inequalities and monotonicity for the ration of $k$ gamma functions," Scientia Magna, vol. 6, no. 1, pp. 40-45, 2010.

[23] F. Merovci, "Power product inequalities for the $\Gamma_{k}$ function," International Journal of Mathematical Analysis, vol. 4, no. 21-24, pp. 1007-1012, 2010.

[24] M. Mansour, "Determining the $k$-generalized gamma function $\Gamma_{k}(x)$ by functional equations," International Journal of Contemporary Mathematical Sciences, vol. 4, no. 21-24, pp. 1037-1042, 2009.

[25] S. Mubeen and G. M. Habibullah, " $k$-fractional integrals and application," International Journal of Contemporary Mathematical Sciences, vol. 7, no. 1-4, pp. 89-94, 2012.

[26] S. Mubeen and G. M. Habibullah, "An integral representation of some $k$-hypergeometric functions," International Mathematical Forum, vol. 7, no. 1-4, pp. 203-207, 2012.

[27] S. Mubeen, "Solution of some integral equations involving conuent $k$-hypergeometric functions," Journal of Applied Mathematics, vol. 4, no. 7, pp. 9-11, 2013. 


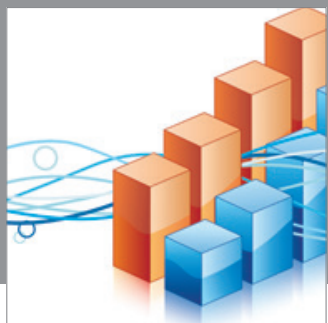

Advances in

Operations Research

mansans

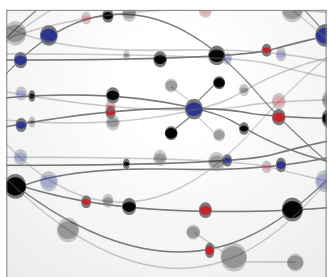

The Scientific World Journal
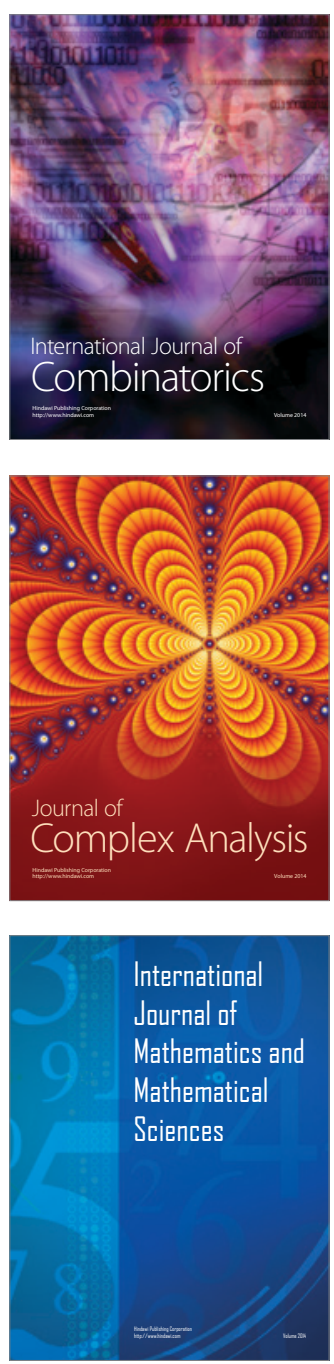
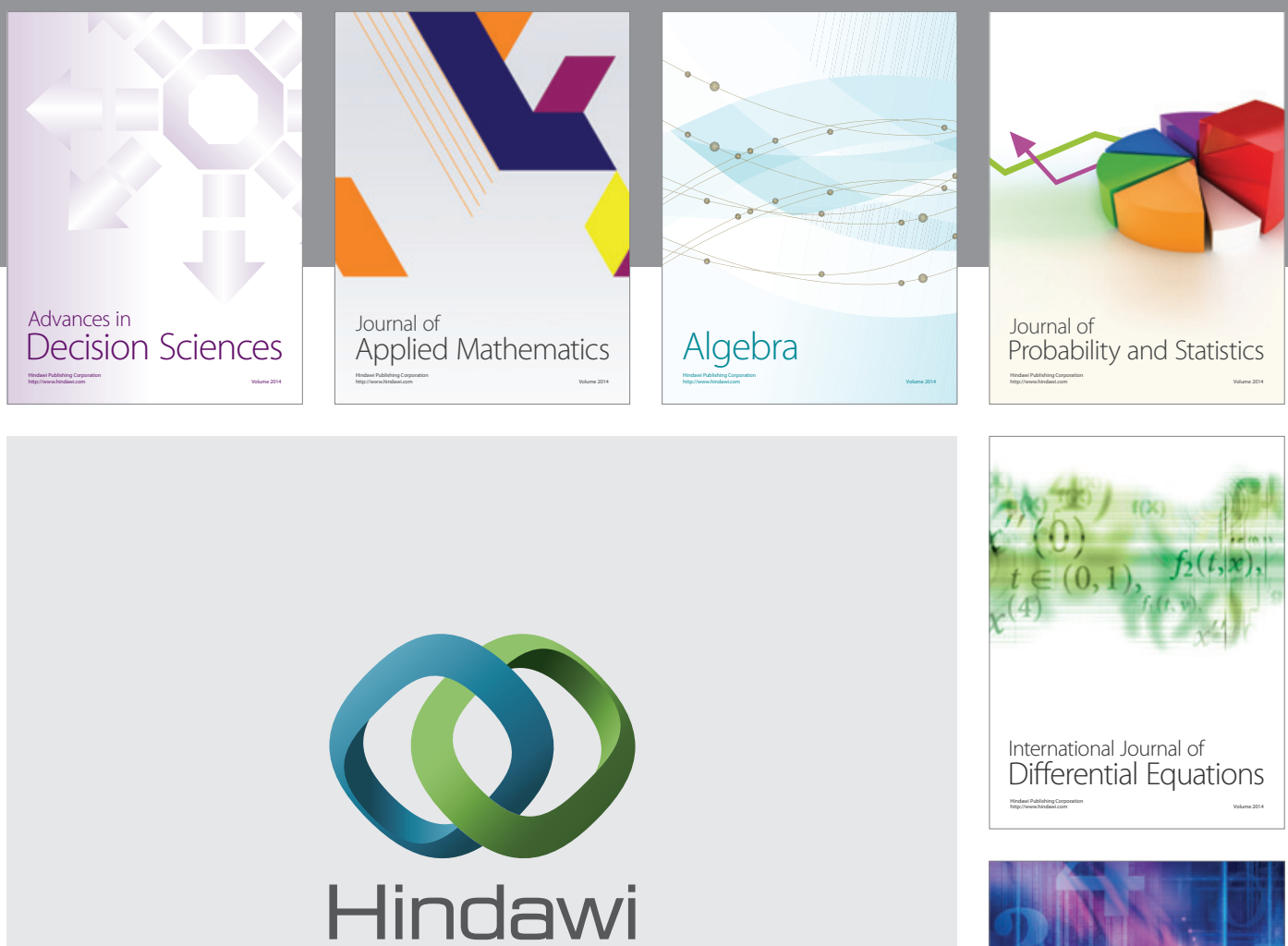

Submit your manuscripts at http://www.hindawi.com
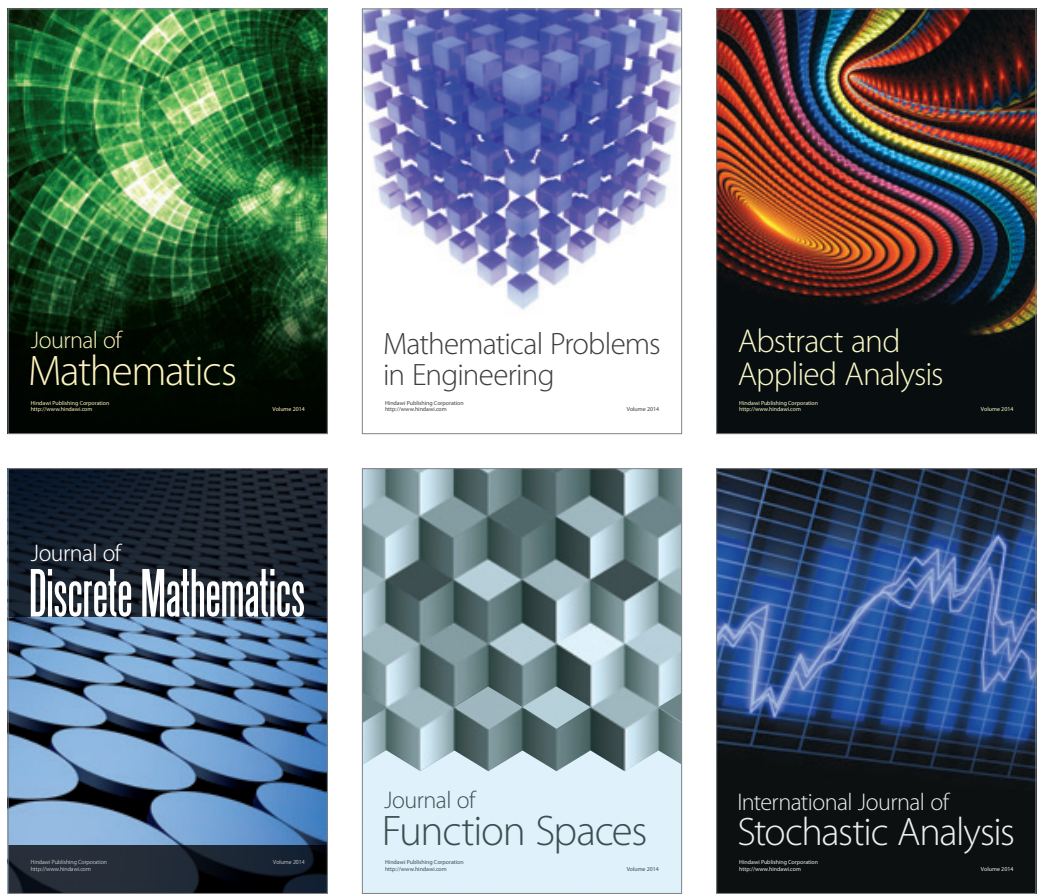

Journal of

Function Spaces

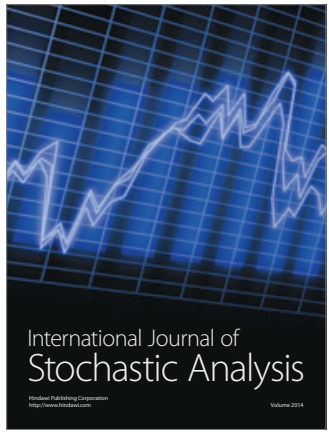

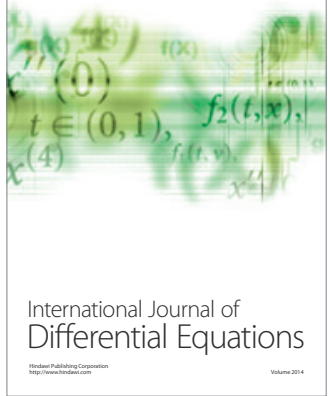
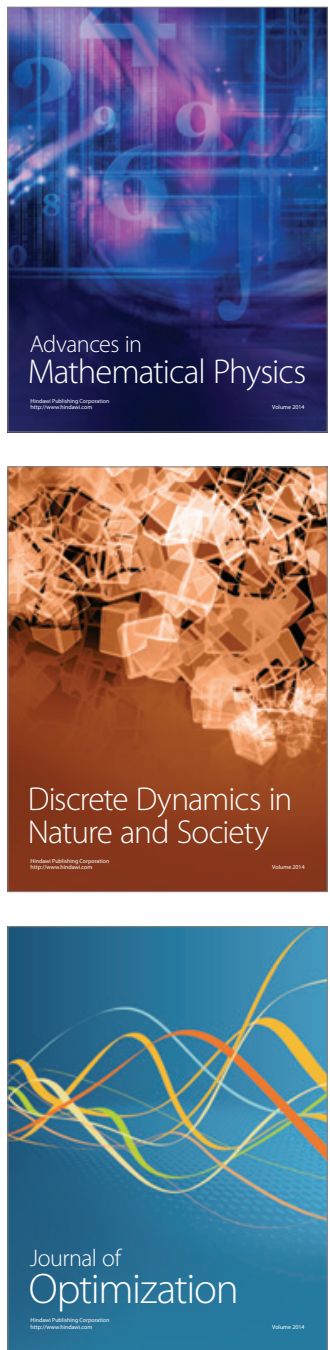